\title{
Wind accretion in binary stars
}

\section{Angular momentum loss}

\author{
B. Jahanara ${ }^{1}$, M. Mitsumoto ${ }^{1}$, K. Oka ${ }^{2}$, T. Matsuda ${ }^{1}$, I. Hachisu ${ }^{3}$, and H. M. J. Boffin ${ }^{4}$ \\ 1 Department of Earth and Planetary Sciences, Kobe University, Rokko-dai 1-1, Nada-ku, Kobe 657-8501, Japan \\ e-mail: [jahanara;meg]@jet.scitec.kobe-u.ac.jp; tmatsuda@kobe-u.ac.jp \\ 2 Mizuho Information and Research Institute, Inc., Kanda-Nishikicho 2-3, Chiyoda-ku, Tokyo 101-8443, Japan \\ 3 Department of Earth Science and Astronomy, College of Arts and Sciences, University of Tokyo, Komaba 3-8-1, Meguro-ku, \\ Tokyo 153-8902, Japan \\ e-mail: hachisu@chianti.c.u-tokyo.ac.jp \\ ${ }^{4}$ European Southern Observatory, Karl-Schwarzschild-Str. 2, 85738 Garching, Germany \\ e-mail: hboffin@eso.org
}

Received 7 February 2005 / Accepted 11 May 2005

\begin{abstract}
We present three-dimensional hydrodynamic calculations of mass transfer in an interacting binary system in which one component undergoes mass loss through a wind, and does so for various values of the mass ratio. The radius of the masslosing star is taken to be half the size of its Roche lobe. Calculations are performed for gases with a ratio of specific heats $\gamma=5 / 3$. Mass loss is assumed to be mechanically, thermally, or radiatively driven.

We compute the specific angular momentum of gas escaping the system $\left(l_{w}\right)$ for these various cases. We show that $l_{w}$ does not reach a value higher than $\sim 1.2$ for very low wind velocities and that it reaches the limiting case of a spherically symmetric wind for large wind velocities, for mass ratio smaller or equal to 1 . For larger mass ratio, however, $l_{w}$ is larger than the expected limiting value. The value of $l_{w}$ depends slightly on the wind mechanism which modifies the relation between the wind velocity at the surface of the star and the velocity at the Roche lobe surface.

The specific angular momentum, $l_{w}$, is large enough in a wide range of velocities to imply a shrinking of the system. This makes the symbiotic channel for Type Ia supernovae a plausible one and could also help explain the existence of Barium stars and other Peculiar Red Giants with orbital periods below, say, 1000 days.
\end{abstract}

Key words. hydrodynamics - binaries: close - binaries: symbiotic - supernovae: general - accretion, accretion disks

\section{Introduction}

The stellar components of a binary system can interact in multiple ways, which must be understood in order to account for the existence of a wide variety of systems and phenomena. The stars can, for example, experience tidal interactions or mass loss and mass transfer. If the system is close enough and one of the two components fills its Roche lobe, mass will be transferred through the inner Lagrangian point towards the massaccreting companion. If the latter is large enough, it will accrete directly the matter as in Algol-type binaries, while if it is too small, as is the case for e.g. accreting white dwarf in cataclysmic variables, infalling matter will form a disc around the object.

Another possibility of interaction arises when none of the stars fills its Roche lobe, but one of them is losing mass through a stellar wind, a generic feature of stars in many evolutionary stages. Stellar winds are the strongest for giant or supergiant stars and for massive blue stars. Mass transfer by wind will thus play an essential role in the evolution of detached binary systems containing such stars. This includes systems such as symbiotic stars, precursor of peculiar red giants, $\zeta$ Aurigae stars, and massive X-ray binaries.

The mass loss by one of the components can result in a plethora of effects. As part of the mass lost will be captured by the mass-accreting star - see Paper I (Nagae et al. 2004) - this can account for the symbiotic phenomenon (see Mikołajewska 1997, for a review) or for the existence of Peculiar Red Giants (Boffin \& Jorissen 1988; Theuns et al. 1996).

But the mass - and the angular momentum - lost from the system also has a consequence on the orbital elements of the system itself. An isotropic mass loss from the masslosing star will for example lead to an increase in the semimajor axis. This was taken into account in the model for formation of Barium stars by Boffin \& Jorissen (1988) and was shown to be consistent with observations through a statistical analysis of spectroscopic binaries containing red giants (Boffin et al. 1993). When the angular momentum loss is large enough, 
however, Hachisu et al. (1999, hereafter HKN99) showed that the system can shrink, thereby offering a wider channel for symbiotic systems to become Type Ia supernovae. It is thus essential to precisely derive the angular momentum loss of a binary system to account for its future evolution. This is the goal of the present paper.

Nagae et al. (2004, hereafter Paper I) performed threedimensional hydrodynamic calculations in order to investigate mass transfer through wind in a close binary system with a mass ratio of one. They characterized the flow by the mean normal velocity of wind on the critical Roche surface of the mass-losing star, $V_{\mathrm{R}}$. When $V_{\mathrm{R}}<0.4 A \Omega$, where $A$ and $\Omega$ are the separation between the two stars and the angular orbital velocity of the binary, respectively, they obtained Roche-lobe over-flow (RLOF), while for $V_{\mathrm{R}}>0.7 A \Omega$ they observed wind accretion. Very complex flow patterns in between these two extreme cases were found. They also found that mass accreted by the compact star is roughly a factor 5 smaller than what would be naively deduced from the Bondi-Hoyle-Lyttleton formalism.

Our main goal is to study the amount of specific angular momentum carried by the gas escaping from the system. In this paper, we will pursue this analysis by performing simulations for three different mass ratios and three wind mechanisms.

\section{Assumptions and numerical method}

\subsection{Model}

We consider a detached binary system consisting of one star of mass $M_{2}$ ejecting gas as a stellar wind, while the other, more compact, component of mass $M_{1}$ partly accretes the gas. We define the mass ratio as $q=M_{2} / M_{1}$ and study the cases of $q=1 / 3,1$ and 3 . We normalize the length by $A$, and the time by $1 / \Omega$. The surface of the mass-losing star is assumed to be an equi-potential surface, whose mean radius is half its Roche lobe radius. The radius of the mass accreting star is assumed to be 0.014 .

We assume that the gas is an ideal one and is characterized by the ratio of specific heats $\gamma$. In the present work we consider the case of $\gamma=5 / 3$. We neglect other complex effects such as magnetic fields, radiation, and viscosity (except for the numerical one).

\subsection{Method of calculation}

We solve the three-dimensional Euler equations using a finite volume scheme. As Riemann solver, we use the SFS scheme and apply the MUSCL method to interpolate physical variables in a cell; the details of the scheme are described in Makita et al. (2000), and RLOF simulations using the same method are given in Fujiwara et al. (2001).

Calculations are done in the rotating frame of a binary and include the gravity of the two stars (except in the radiative wind case where we suppress the gravity of the mass-losing star), the centrifugal and Coriolis forces. We use a Cartesian coordinate with the origin at the mass accreting star. The computational region is $-3.5<x<2.5,-3.0<y<3.0,0<z<2.5$, and we assume symmetry around the orbital plane. The region is divided into $217 \times 217 \times 91$ cells. The mass-accreting star is represented by a single cell whose dimensions are thus 0.0278 in our dimensionless units.

\subsection{Wind mechanisms, boundary conditions, and initial condition}

From an OB star, stellar wind is mainly accelerated by line absorption of UV radiation. In symbiotic stars or in precursors of peculiar red giants (PPRGs), the stellar wind mechanism from the cool star is poorly known, and may imply radiation onto dust grains. One needs, however, to still bring the matter far enough away from the star so that dust grains can form. In more evolved red giants, this may be done through the effect of pulsation. The mechanism operating in non-pulsating red giants, as they seem to exist in some symbiotic or extrinsic S stars, is still far from being fully understood.

It is therefore clear that simplifying assumptions have to be made. In the present study, we have considered three cases for the wind mechanisms: mechanically driven, thermal driven, and radiation pressure driven stellar wind. For the mechanical driven wind, the wind has a given normal velocity in addition to its thermal one, at the surface of the mass-losing star. We fix the sound speed of gas at the surface of the mass-losing star to be $C_{\mathrm{s}}=0.5$, and vary the given normal velocity of ejected gas at the mass-losing star as $V=0.5-4$.

In the thermal driven case, we set $V=0.5$ and vary the sound speed as $C_{\mathrm{s}}=0.5-4$. Thus if the pressure inside the mass-losing star gets larger than that outside the mass-losing star, the gas acquires a higher velocity. The velocity of the ejected gas is computed by solving a Riemann problem between the two states. This is similar to the solar wind type and to what was done in Paper I. In both these cases, the full gravitational potential of the stars is taken into account.

In the radiative driven case, we assume that the gas is accelerated by some mechanism, to reproduce mass loss in red giants and hot blue stars. Here we assume this acceleration to exactly balance the gravitational force of the mass-losing star. We inject the gas as in the mechanically driven case by setting $C_{\mathrm{S}}=0.5$ and $V=0-4$ at the surface of the mass-losing star. This is somewhat similar to e.g. the simulations by Theuns et al. (1996).

The outer parts of the mass-losing star is filled by a gas of density $\rho=1$. The sound speed of the gas on the outer parts depends on the model under study as mentioned above. At $t=0$ the computational region, except the mass-losing star, is filled by a tenuous gas with density $\rho_{0}=10^{-5}$, velocity $u_{0}=v_{0}=$ $w_{0}=0$, and pressure $p_{0}=10^{-4} / \gamma$. The computational region is gradually filled with the gas ejected from the mass-losing star.

The outside of the computational region is filled by the initial gas all the time. Mass outflow/inflow from the outer boundary can occur; the amount of outflow/inflow is also calculated by solving Riemann problems between two states. This (outer) boundary condition, which we call the ambient condition, insures the stability of the computation. The inside of the mass accreting hole is almost vacuum and the gas approaching the hole is absorbed. 
We start the calculation at $t=0$ and run it for 5 orbital periods. This is enough to reach a steady state. Calculations are done by using either 8 CPUs of the NEC SX-6 vector supercomputer or 8 CPUs of a Sun Fire 12K with UltraSPARC III $\mathrm{Cu} 1.05-\mathrm{GHz}$. One run takes one hour on the SX-6, while it takes one day on the Sun Fire 12K. In both cases, a shared memory mode is used. We compute 89 cases in total.

\subsection{Calculation of the specific angular momentum}

The specific angular momentum is defined as:

$l_{w}=x^{2}+y^{2}+x v-y u$,

where the origin of $x$ and $y$ is at the centre of gravity in this formula, and $(u, v)$ are the $x$ and $y$ component of the wind velocity. The first and the second term of the equation represent the angular momentum due to the rotation of the frame. In order to calculate the mean specific angular momentum, we use the following formula

$\left\langle l_{w}\right\rangle=\frac{\mathrm{d} J / \mathrm{d} t}{\mathrm{~d} M / \mathrm{d} t}$,

where $\mathrm{d} J / \mathrm{d} t$ and $\mathrm{d} M / \mathrm{d} t$ are the total angular momentum loss rate and the total mass loss rate from the binary system, and they are calculated on the surface of a cubic box, which is suffciently large but does not coincide with the outer boundary. This choice is made in order to avoid unnecessary boundary effects.

\section{Numerical results}

\subsection{Flow patterns}

As was done in Paper I, for each simulation we calculate the mean value of the normal component of the speed of gas on the critical Roche surface $V_{\mathrm{R}}$. This allows a better comparison between the different simulations. As was shown in Paper I, the flow pattern depends heavily on the value of $V_{\mathrm{R}}$. In the case of mass ratio unity, when $V_{\mathrm{R}}<0.4$, we have a Roche lobe overflow type of flow (RLOF), while if $V_{R}>0.7$, we have a wind accretion flow. In between, we have very complicated flows.

In Fig. 1, we show the relation between the normal velocity on the surface of the mass-losing star $V$ and the normal velocity on the critical Roche surface $V_{\mathrm{R}}$ in our simulations, for three values of the mass ratio. A clear distinction is seen between RLOF flows with very low $V_{\mathrm{R}}$ values and wind type accretion flows with large $V_{\mathrm{R}}$.

As a complement to the flow patterns shown in Paper I, we show here, as an example of the flow pattern, some numerical simulations when the mass-losing star is the more massive, i.e. $q=3$, for three values of the initial gas velocity, in the mechanical driven case.

Figure 2 shows the density contours on the orbital plane for the model with $q=3, V=1$, and $V_{\mathrm{R}}=0.034$, which bears some resemblance to RLOF type flows. No accretion disc is observed, as our $\gamma$ index is $5 / 3$ and no cooling mechanism operates. The streamlines corresponding to this flow are also

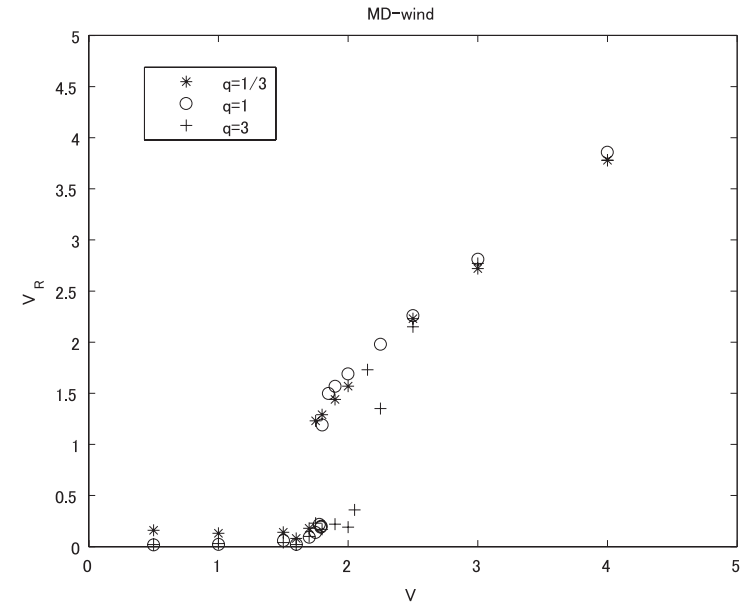

Fig. 1. Normal velocity on the critical Roche surface $V_{\mathrm{R}}$, as a function of the normal velocity on the surface of the mass-losing star $V$ for three mass ratios $q=1 / 3,1$, and 3 , in the case of a mechanical driven wind.

shown in Fig. 2. The flow pattern is very complicated and is the result of the collisions between many different streams.

Figure 3 shows the flow when the velocity is increased, such that $V=1.9$, and $V_{\mathrm{R}}=0.22$. In this case, the wide spiral seen in the lower velocity run has sensibly disappeared, and we are left with an accretion cone, which is, however, still very deformed in a spiral shape.

With further increase of the wind velocity, so that $V=4$, $V_{\mathrm{R}}=3.78$, as shown in Fig. 4, the flow becomes very typical of a Bondi \& Hoyle accretion flow. In these three simulations, the specific angular momentum loss $l_{w}$ was $1.02,0.57$, and 0.15 , respectively.

In Fig. 5, we compare two simulations for mass ratio unity and $V=0.5, C_{\mathrm{S}}=0.5$. On the one side, we compute the case of a thermally driven flow. In this case the gravity of the masslosing star is fully taken into account, and the wind velocity at the surface of the Roche lobe is $V_{R}=0.03$. The other run deals with a radiative driven wind, in which the gravity due to the mass-losing star is totally compensated for by the radiation pressure. In this case we have $V_{\mathrm{R}}=0.83$. This again illustrates that the different wind mechanisms produce different values of $V_{\mathrm{R}}$ for a given value of the velocity at the surface of the star. Then, as the wind velocity at the Roche lobe is different, the flow patterns also differ significantly. This difference has an incidence on the specific angular momentum loss: we compute a value of $l_{w}=1.06$ and 0.43 for the thermally and radiation driven wind, respectively.

\subsection{Angular momentum loss}

For all our simulations, we have calculated the specific angular momentum loss from the system. The results are shown in Figs. 6 and 7. In Fig. 6, we show our results for the three different values of the mass ratio $q=1 / 3,1$, and 3 , in the case of the mechanical driven wind. It can be seen that for low velocity at the surface of the mass-losing star - and hence very low value at the Roche lobe - the specific angular momentum does not vary greatly as a function of the mass ratio. For larger velocities, 

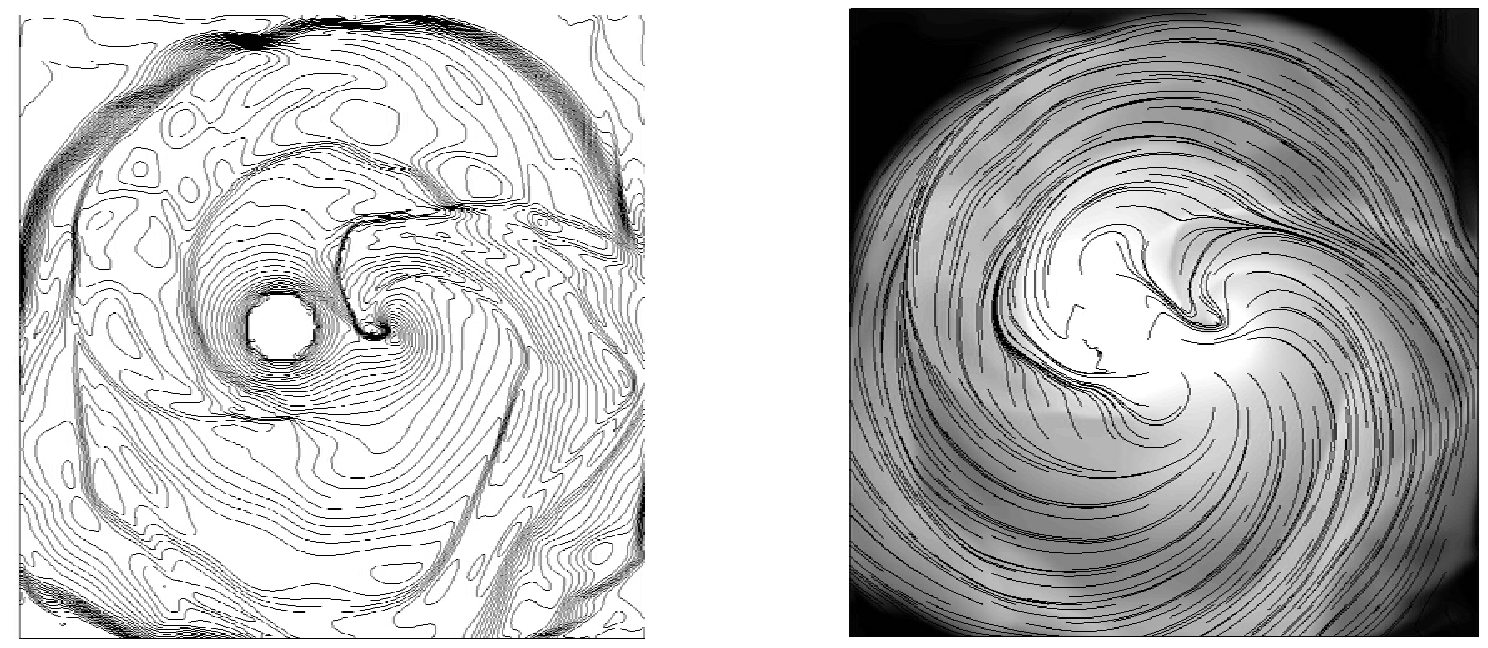

Fig. 2. Left: iso-density curves on the rotational plane of the RLOF type flow for the model with mass ratio $q=3$ and for a mechanically driven wind. In this model $V=1, V_{\mathrm{R}}=0.034, C \mathrm{~s}=0.5$, and $\gamma=5 / 3$. The left circle indicates the mass-losing companion star with radius 0.25 , and the right black dot represents the mass accreting star. Right: streamlines of the flow pattern.

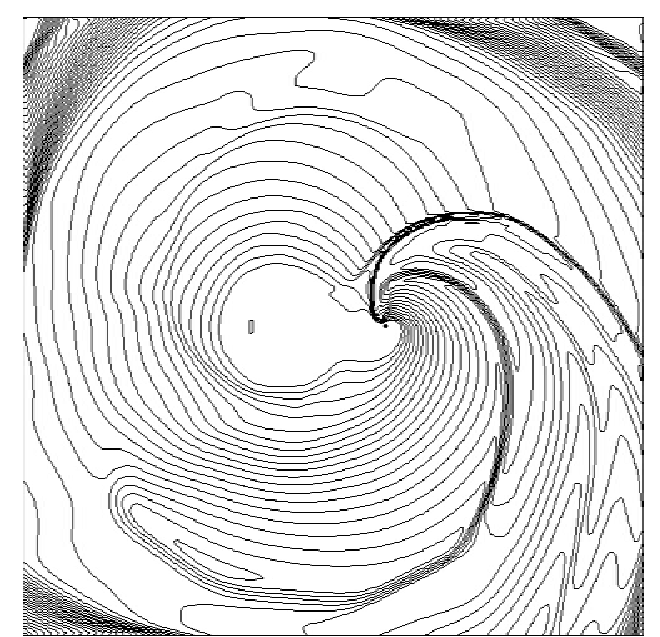

Fig. 3. Same as Fig. 2 except that $V=1.9\left(V_{\mathrm{R}}=0.22\right)$. Near the mass accreting star two spiral-shaped shocks join together.

the values differ substantially, as should be expected. In this case indeed, one can expect that the specific angular momentum loss will simply follow the relation $l_{w}=l_{w, s}=1 /(1+q)^{2}$, easily derivable from assuming that all the mass lost carries the angular momentum of the mass-losing star. The value we obtain is indeed in close agreement with this value for $q=1 / 3$ and 1 , but the value we derive for $q=3$ for large velocities is larger than this value by a factor 2 . We also ran a simulation with a wind velocity of $V=10, V_{\mathrm{R}}=9.98$ for the mass ratio $q=3$ case, which again provided a larger value than $l_{w, s}$ by a factor 2 .

We obtain the following fit for the specific angular momentum loss :

$$
\begin{array}{ll}
l=0.25+\frac{0.12}{V_{\mathrm{R}}+0.02} & \text { for } q=1 \\
l=0.12+\frac{0.12}{V_{\mathrm{R}}+0.02} & \text { for } q=3 .
\end{array}
$$

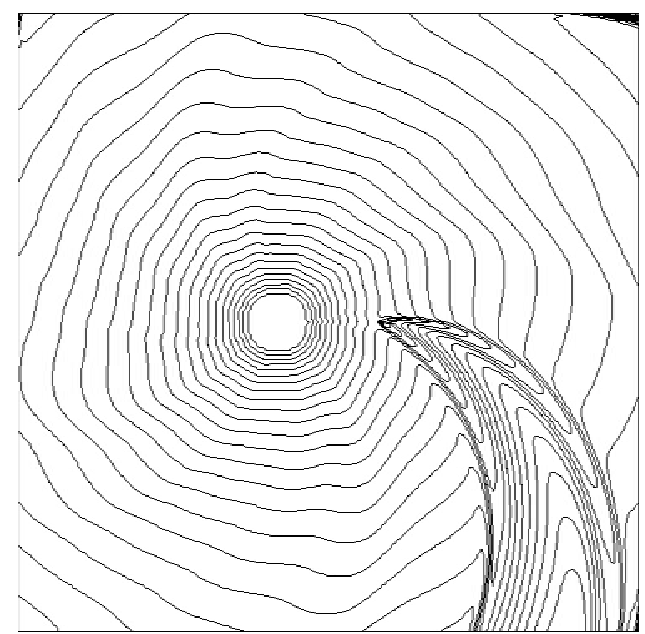

Fig. 4. Same as Fig. 2 but for $V=4\left(V_{\mathrm{R}}=3.78\right)$. This is a typical wind accretion flow pattern with a conical bow shock attached to the mass-accreting star.

It thus appears that if the limiting value for $q=1$ is in agreement with the limiting value given by $l_{w, s}$, the value for $q=3$ is about twice too large.

Figure 7 shows the specific angular momentum loss for $q=1$ and the three different wind mechanisms we studied. It is clear from the right panel that the value of $l_{w}$ does not crucially depend on the wind mechanism - the main effect of the wind mechanism being the relation between the wind at the surface of the star and the velocity at the Roche lobe surface. For the radiative driven wind, however, we note that even for very low velocity on the surface of the star, the specific angular momentum does not go above the value 0.6.

In order to see to what extent our results are sensitive to the radius of the mass losing star, we performed some additional simulations, where the mass-losing star has a radius of $2 / 3$ or $3 / 4$ the Roche lobe radius. We observe that for large velocities, the angular momentum loss increases when the radius of the mass-losing star increases. This is in agreement with the 

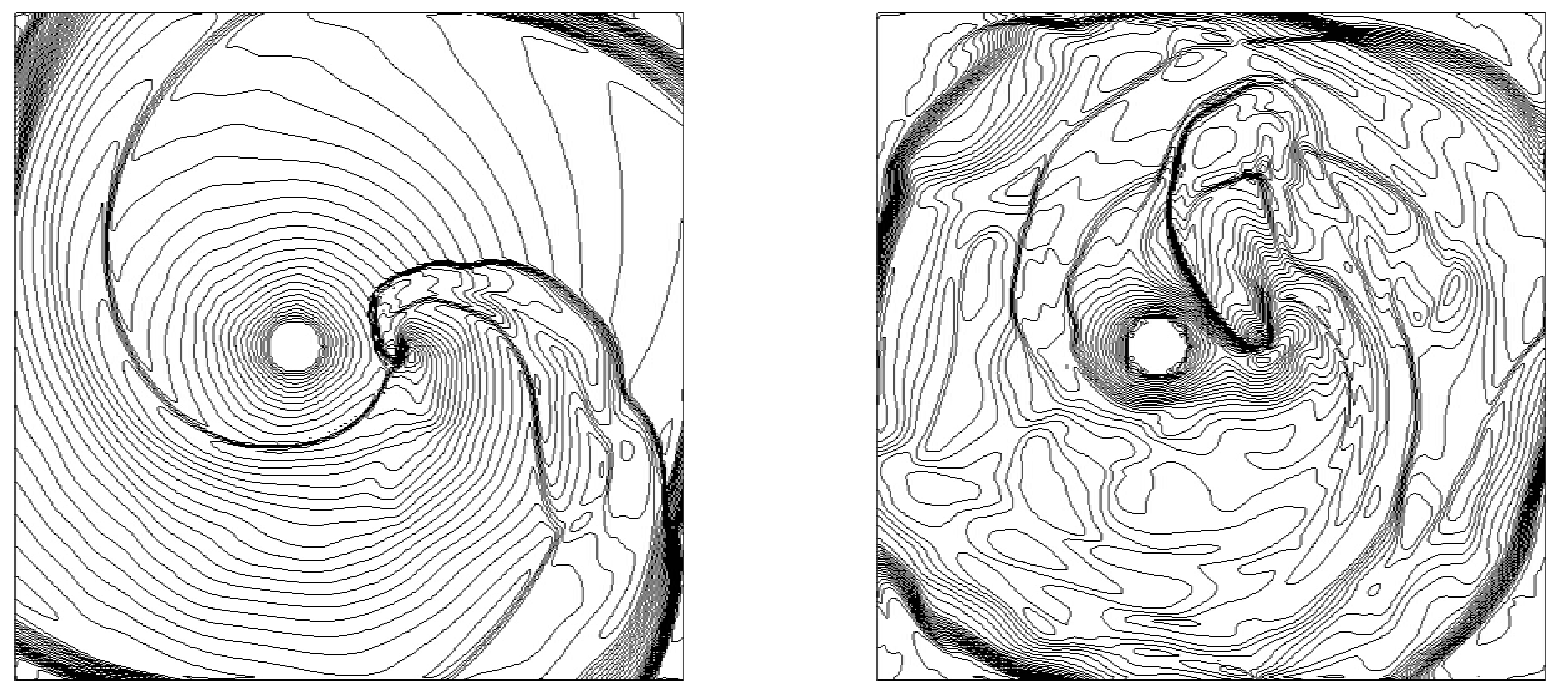

Fig. 5. Comparison between two wind mechanisms: iso-density curves on the rotational plane for models with $q=1, V=0.5, C \mathrm{~s}=0.5$ are shown. Left panel: radiation driven wind. In this model $V_{\mathrm{R}}=0.83$. Right panel: thermally driven wind. In this model $V_{\mathrm{R}}=0.03$. Complicated intermediate type flow in the vicinity of the mass accreting star is observed.
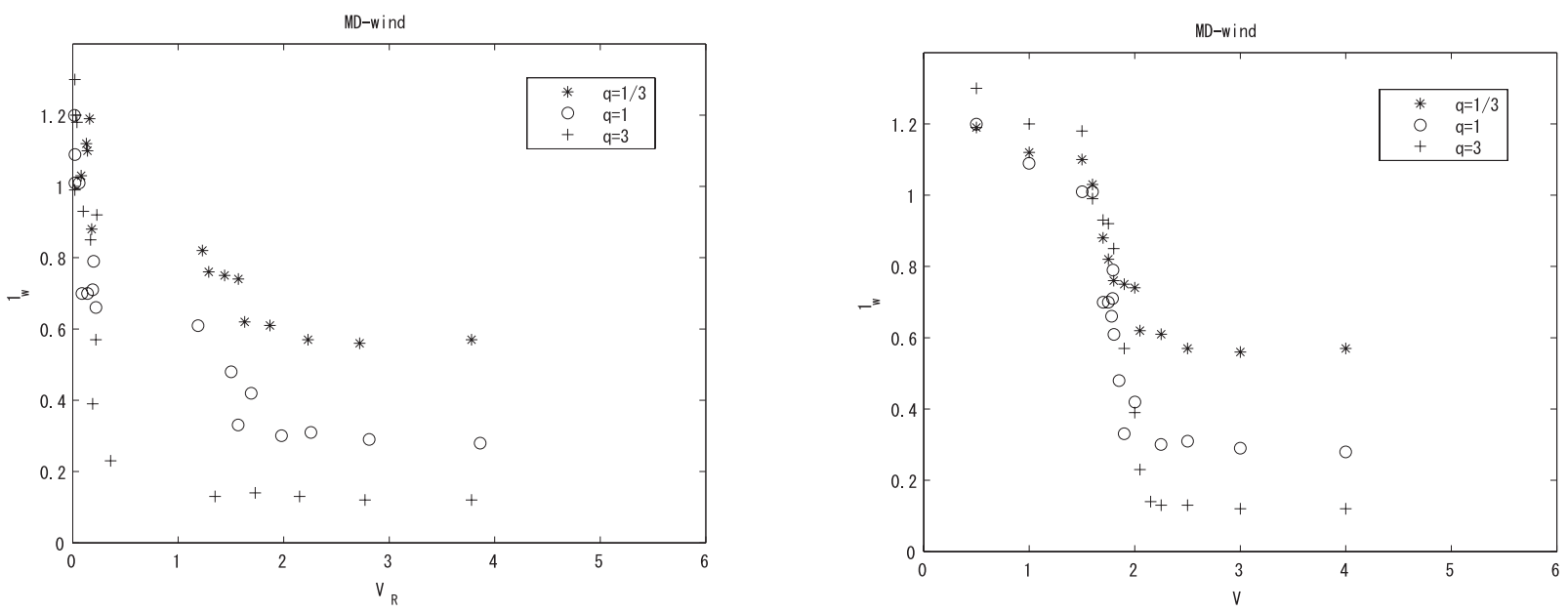

Fig. 6. Left panel: the specific angular momentum loss $l_{w}$ is plotted against the outflowing velocity on the critical Roche surface $V_{\mathrm{R}}$ for the mechanically driven wind. Three mass ratios, $q=1 / 3,1$, and 3 , are examined. The specific angular momentum loss $l_{w}$ decreases from about $1-1.2$ at $V_{\mathrm{R}}<0.5$ (RLOF) to $0.1-0.5$ at $V_{\mathrm{R}}>2$ (wind type flow). For very fast wind, i.e., when $V_{\mathrm{R}}>2$, all the cases approach an asymptotic value. Right panel: the specific angular momentum loss $l_{w}$ is now shown as a function of the outflowing velocity on the surface of the masslosing star, $V$.

ballistic results of Brookshaw \& Tavani (1993). The changes are, however, only about $20 \%$. Still, they confirm the tendency for the angular momentum loss to be larger than the generally used $l_{w, s}$ value. For low velocities, the effect of changing the radius of the mass-losing star is rather small. This is comprehensible due to the fact that at low velocities, gas first needs to leave the Roche lobe anyway in the vicinity of the inner Lagrangian point.

\section{Discussion}

Hachisu et al. (1999) propose a new channel to Type Ia supernovae based on a symbiotic binary system consisting of a white dwarf and a mass-losing red giant. The white dwarf accretes part of the wind from the giant and reaches the Chandrasekhar limit. An essential part of the validity of this channel resides in the fact that the required close white dwarf - red giant binary can form from a very wide binary. They reach their conclusion after noticing that a system where one of the components has a wind whose velocity is smaller or on the order of the orbital velocity takes away the orbital angular momentum effectively, hence shrinking the orbit.

To see this, we can write the variation of the semi-major axis $\dot{a}$ as a function of the angular momentum loss $\dot{J}$ :

$\frac{\dot{a}}{a}=2 \frac{\dot{J}}{J}+\frac{\dot{M}_{1}+\dot{M}_{2}}{M_{1}+M_{2}}-2 \frac{\dot{M}_{1}}{M_{1}}-2 \frac{\dot{M}_{2}}{M_{2}}$

where $\dot{M}_{2}$ and $\dot{M}_{1}$ are the mass loss and accreted, respectively. Writing the angular momentum loss, $\dot{J}$, in function of the dimensionless specific angular momentum loss, $l_{w}$, i.e. 

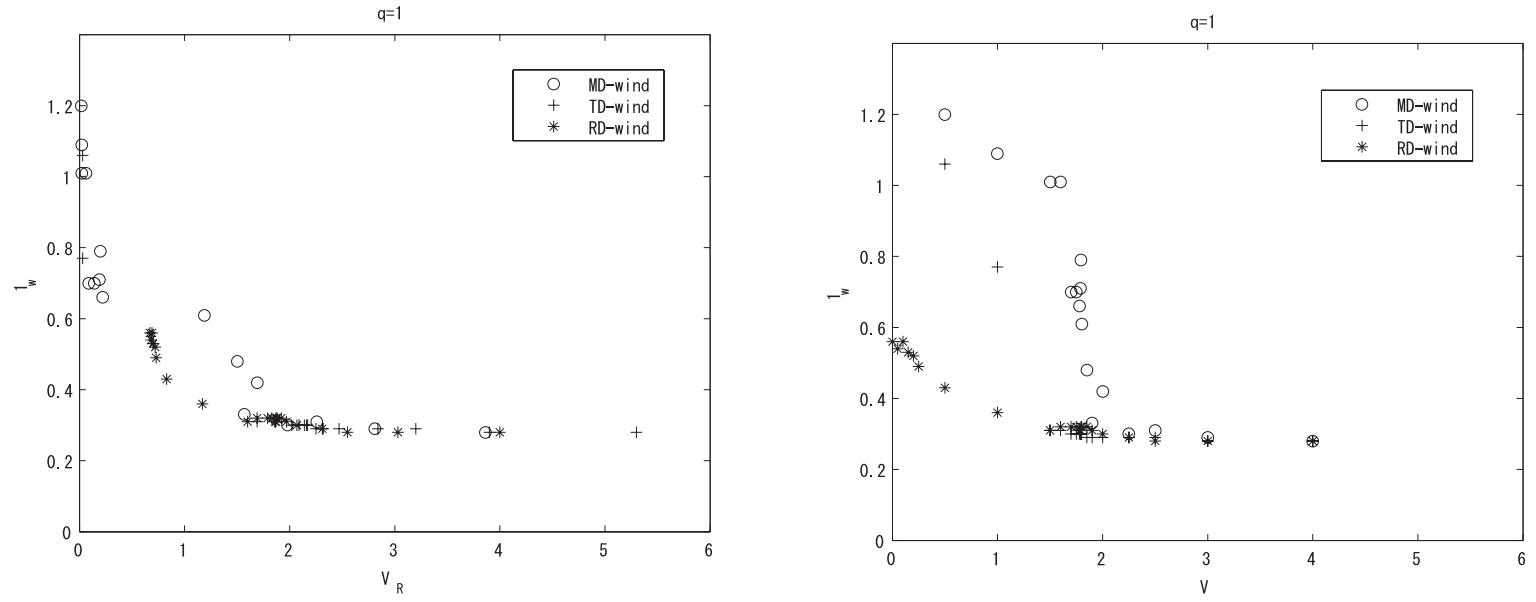

Fig. 7. Left panel: the specific angular momentum loss plotted against the outflowing velocity on the critical Roche surface $V_{\mathrm{R}}$ for $q=1$ and three types of wind mechanisms. The trend is essentially the same for the three different mechanisms except for the radiation-driven wind in the low velocity region. Right panel: the specific angular momentum loss as a function of the outflowing wind velocity $V$ on the surface of the mass-losing star.

$\dot{J}=l_{w} a^{2} \Omega\left(\dot{M}_{1}+\dot{M}_{2}\right)$, we have:

$$
\begin{aligned}
\frac{\dot{a}}{a}= & \frac{\dot{M}_{1}+\dot{M}_{2}}{M_{1}+M_{2}}+2 \frac{\dot{M}_{2}}{M_{2}}\left(l_{w}-1+l_{w} \frac{M_{2}}{M_{1}}\right) \\
& +2 \frac{\dot{M}_{1}}{M_{1}}\left(l_{w}-1+l_{w} \frac{M_{1}}{M_{2}}\right) .
\end{aligned}
$$

Neglecting the mass accreted, which was shown in Paper I to be very small in most cases, we have

$\frac{\dot{a}}{a}=\left(\frac{M_{2}}{M_{1}+M_{2}}+2 \frac{M_{1}+M_{2}}{M_{1}} l_{w}-2\right) \frac{\dot{M}_{2}}{M_{2}}$.

HKN99 then note that with a value of $l_{w} \sim 1$, the function in front of $\dot{M}_{2} / M_{2}$ would be positive, hence the orbit would shrink, because $\dot{M}_{2}<0$. They then arrive at the conclusion that $l_{w} \sim 1$ is a plausible value for low wind speed flows by computing the relation between the wind velocity at the Roche lobe surface and $l_{w}$ by calculating many orbits of test particles moving under the Roche potential and Coriolis force. They obtained the relation $l_{w} \simeq 1.7-0.55 v_{R}^{2}$ for small velocities, where the limiting case $l_{w}=1.7$ for zero wind speed was obtained by Sawada et al. (1984) for a two-dimensional hydrodynamic simulation. Our results show, however, that the maximum value of $l_{w}$ is closer to 1.2 and not as large as 1.7.

Brookshaw \& Tavani (1993) also performed ballistic calculations of wind-driven mass loss in a binary system. They mainly considered the case where the mass-losing star is filling its Roche lobe and obtained large angular momentum losses. For large velocities, they obtained in the $q=1$ case values five times larger than the predicted asymptotic value. However, the discrepancy became less severe when they studied the case with the secondary well inside their Roche lobe, which is closer to our study. In this case, for large velocities, they obtained the expected asymptotic value. They also appreciate that the ballistic nature of their calculations only describes high velocity flows well and that "a complete treatment of binary angular momentum loss requires a hydrodynamical treatment of low mass velocity mass outflows". This is exactly what is presented in the present paper.

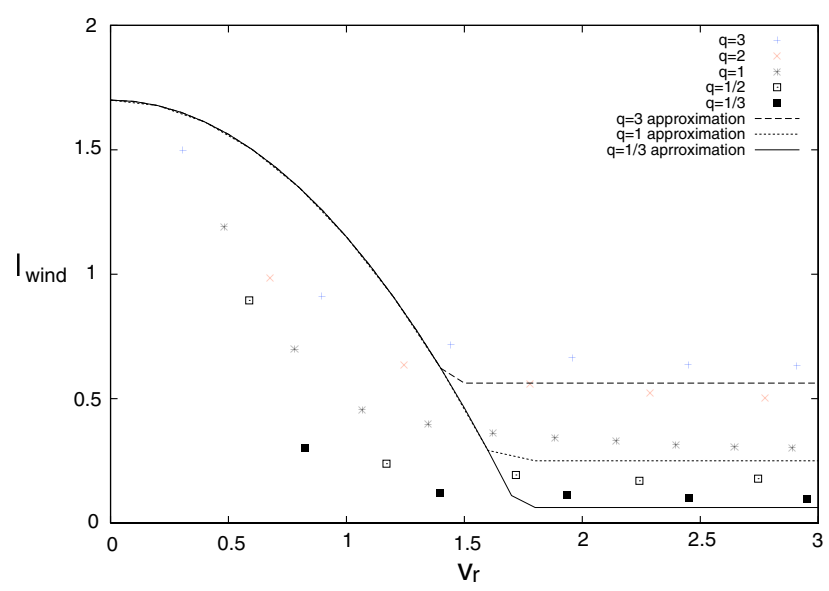

Fig. 8. Specific angular momentum loss of the wind plotted against the outflowing velocity near the inner critical Roche lobe. It was calculated using test particles moving under the Roche potential and Coriolis force. The points indicate our results, while the curves are the fits obtained by Hachisu et al. (1999). Note the discrepancy due to a difference in the averaging method. Our results are in good agreement with the results of our three-dimensional hydrodynamic simulations (Yamamoto 2004).

We also redid the test particles calculations (Yamamoto 2004) and the result is shown in Fig. 8. It can be seen that these values are in agreement with the more accurate values obtained with our three-dimensional hydrodynamical simulations. The lines in the figure represent the fit used by HKN99. It can be seen that these fits do not follow our points and we believe the reason lies in the way the averaging of the specific angular momentum is done. As noted by HKN99, the correct average should include the mass-loss rate as $v_{\mathrm{o}} \sin \theta_{i} \Delta \theta \Delta \Phi / 4 \pi$, but it seems that these authors did not finally take this into account.

It is thus important to see if our new results imply a revision of the conclusions of Hachisu et al. For this, we rewrite Eq. (6) as

$\frac{\dot{a}}{a}=L(q) \frac{\dot{M}_{2}}{M_{2}}$ 
where $L(q)=q /(1+q)+2 l_{w}(1+q)-2$, and note that for the system to shrink we need to have $L(q)>0$ (as $\dot{M}_{2}$ is negative) and, hence, $l_{w}>0.5(2+q) /(1+q)^{2}$. For the mass ratio of 7 considered by HKN99, one thus only needs $l_{w}>0.07$, which is shown to be possible for low wind velocity in our simulations. We have therefore run four simulations with $q=7$ in the radiative driven wind for four values of the wind velocity, resulting in specific angular momentum loss of $0.13,0.11,0.10$, and 0.10 , for $V=0,1,2,10$ and $V_{\mathrm{R}}=0.7,1.17,2.06$, and 9.92, respectively. Thus, such a system would indeed shrink for a large range of wind velocities. And here again, the value we derive for very large velocities is larger than the simple limiting case, by a factor 5 at least.

We note that, in the limiting case of a spherically symmetric wind that does not interact with the mass-accreting star, we have $l_{w}=1 /(1+q)^{2}$, and the system is thus always widening. However, as noted above, for large mass ratio, the specific angular momentum loss is always larger than this limiting case the difference increasing with the mass ratio - with the possible result that the above conclusion is contradicted.

From the results of our numerical simulations, it appears that the system would shrink - when assuming no mass accretion - when the wind speed at the Roche lobe is smaller than roughly between 0.7 and 1.7 times the orbital velocity. One can also show that the conclusion still holds, when the mass ratio is larger or equal to one, when mass accretion is taken into account.

The simulations with $q=3$ even show that the specific angular momentum loss for large velocities is rather large. In this case, it is easy to have the system shrink. This could have important consequences for the progenitors of barium stars, for example.

The most widely adopted model for the formation of peculiar red giants, Barium, $\mathrm{CH}$, and $\mathrm{S}$ stars, require a binary system containing an Asymptotic Giant Branch star (AGB) transferring mass via its stellar wind to a main sequence companion which becomes polluted in Carbon and s-process elements (see e.g. Boffin \& Jorissen 1988). The AGB then evolves into a white dwarf while the companion will appear as Carbon or s-process rich and, when on the giant branch, as a peculiar red giant. Boffin \& Zacs (1994) have shown that an accretion efficiency of only a few percent is enough to explain present Barium stars. This model fails, however, to explain most small period barium star systems with orbital period below, say, 1000 days. The existence of such systems was always a problem in the wind accretion theory of Boffin \& Jorissen (1988) since if one takes the value of the specific angular momentum given by the spherically symmetric wind, one finds for e.g. $q=3$ that the system has enlarged by a factor 3 .

If the present system has a period below 1000 days, say, then its present separation is roughly $2.5 \mathrm{AU}$, implying an initial semi-major axis of less than $1 \mathrm{AU}$. This is too small to hope to fit an asymptotic giant branch star without having Roche-lobe overflow. With the new value of the specific angular momentum we obtain, the system did not expand, but instead shrank by a factor 3 to 10 , depending on the ratio of the wind speed to the orbital velocity. It remains, however, to check that this scenario can quantitatively explain the overabundances seen in barium stars by performing an analysis similar to Boffin \& Zacs (1994), but this is deferred to a later paper. We note that Brookshaw \& Tavani (1993) also emphasize the importance of angular momentum loss in the evolution of binary systems and the fact that the often used approximation $l_{w}=l_{w, s}$ can lead to wrong conclusions, in particular in X-ray binaries.

\section{Conclusion}

We have performed three-dimensional hydrodynamic simulations of mass transfer in a binary system when the mass-losing star does not fill its Roche lobe. The simulations were performed for different wind mechanisms and for three mass ratios. The main purpose of the calculations was to compute the specific angular momentum loss from the system as a function of the wind speed on the Roche surface.

We find that the specific angular momentum reaches a maximum value of 1.2 for very low velocities, almost independently of the mass ratio. For greater velocities, the specific angular momentum decreases and converges to a value greater or equal to the expected one, obtained when assuming that all the mass lost carries the angular momentum of the mass-losing star. The discrepancy becomes larger for larger mass ratios, being 2 for $q=3$ and 5 for $q=7$. This has significant consequences for a variety of binary systems, i.e. progenitors of Type Ia supernovae or small period Barium stars.

Acknowledgements. The authors would like to acknowledge Drs. H. Fujiwara and T. Nagae, who developed the present computer code, and Miss M. Yamamoto, who made particle simulations. Calculations were performed on the NEC SX-6 at ISAS/JAXA and also on the Sunfire $12 \mathrm{~K}$ at Kobe University. T.M. was supported by the grant in aid for scientific research of the Japan Society of Promotion of Science (13640241) and I.H. by (11640226). This work was supported by "The 21st Century COE Program of Origin and Evolution of Planetary Systems" in the Ministry of Education, Culture, Sports, Science and Technology (MEXT). H.B. thanks Kobe University for its hospitality.

\section{References}

Boffin, H. M. J., Cerf, N., \& Paulus, G. 1993, A\&A,

Boffin, H. M. J., \& Jorissen, A. 1988, A\&A, 205, 155

Boffin, H. M. J., \& Zàcs, L. 1994, A\&A, 291, 811

Bondi, H., \& Hoyle, F. 1944, MNRAS, 114, 195

Brookshaw, L., \& Tavani, M. 1993, ApJ, 410, 719

Fujiwara, H., Makita, M., Nagae, T., \& Matsuda, T. 2001, Prog. Theor. Phys., 106, 729

Hachisu, I., Kato, M., \& Nomoto, K. 1999, ApJ, 522, 487 (HKN99)

Makita, M., Miyawaki, K., \& Matsuda, T. 2000, MNRAS, 316, 906

Mikołajewska, J. 1997, in Physical Processes in Symbiotic Binaries and Related Systems, ed. J. Mikołajewska (Warsaw: Copernicus Foundation for Polish Astronomy), 3

Nagae, T., Oka, K., Matsuda, T., et al. 2004, A\&A, 419, 335 (Paper I) Sawada, K., Hachisu, I., \& Matsuda, T. 1984, MNRAS, 206, 673

Theuns, T., Boffin, H. M. J., \& Jorissen, A. 1996, MNRAS, 280, 1264 Yamamoto, M. 2004, Bachelor Thesis (in Japanese), Kobe University 